Aus der Königl. Chirurgischen Universitätsklinik und Poliklinik in Königsberg i. Pr. (Direktor: Prof. Dr. Kirschner.l

\title{
Isolierte Tuberkulose der Dura mater spinalis mit totaler Querschnittslähmung.
}

Von Dr. med. Erwin Baumann, Assistenzarzt der Klinik.

(Mit 2 Abbildungen.)

In unscrer K'Klinik kam kürzlich eine isolierte Tuberkulose der Dura mater spinalis zur Beobachtung. Da wir in der uns zugänglichen İiteratur bisher keinen Bericht über cine ,primäre" bzw. isolierte Tuberkulose der harten Rückenmarkshaut finden konnten, so glauben wir, daß die Veröffentlichung des Falles einiges Interesse finden wird.

Frau Gertrud F., 4rjähr., aus K.

Aus der $A$ namnese ergibt sich folgendes: die Mutter starb betagt an IIerzschlag, der Vater mit 72 Jahren an Magenkrebs. ihr Mann an Iferzschlag. l'at. hat 4 gesunde Kinder. Sic selbst ist mit Ausnahme einer Lungenentzundung in jungen Jahren immer gresund gewesen. Familienanamnese auf Tuberkulose negativ. Im Oktober 1916 begannen sich zwischen den Schulterblättern ziehende Schmerzen einzustellen, dic als ,rheumatisch" angesehen wurden. Mit der Zeit nahmen sie aber trotz antirheumatischer Behandlung zu. Vor allem stellten sie sich beim Liegen ein und wurden dabei schlieblich so unerträglich, dals Patientin sitzend auf einem Stuhl schlief. Gegen Weihnachten 1916 breiteten sich die Schmerzen gürtelförmig auf der linken Körperseite bis gegen die Brust hin aus. um daselbst nach I4 Tagen $\%$ verschwinden. Am 27. XII. bemerkte die Kranke zum ersten Male Parästhesien an beiden Füßcn, die ganz. allmählich die Beine hinaufstiegen. Sie waren links inımer höher und heftiger als rechts. Im Verlaufe von nur 2 Tagen stellten sich dann um Neujahr herum auch eine Cefühllosigkeit und Schwäche in bericlen Beinen ein; „sie schliefen allmählich ein und wurden schlaff". so daß3 Patientin nicht mehr gehen konnte. In wenigen Tagen stiegen Gefühllosigkeit und I.ähmung beidseits bis zum unteren Sternalrande und VIII. IBrustwirbel in die Iöhe. Dabei trat zuerst I rinurerhaltung: 
dann Incontinentia vesic. urin. und Verstopfung auf. -... Gegenwärtig verspürt I'atientin nur Schmerzen in der oberen Rückenhältte.

Status: Mittelgroße Frau, in mäBigem Iirnährungszustande. Fettpolster geschwunden. Haut und Schleimhäute sind blaß. Kopt o. B. Innere Organe: vorn über der r. Iungenspitze ist ein leicht abgeschwächter Klopfschall vorhanden; auskultat. o. B. Herz und Gefäßsystem o. B. Bauchorgane mit Ausnahme einer ausgesprochenen Darmträgheit nach Bariummahlzeit o. B. Der Urin geht unwillkürlich ab, ist frei von Eiweils und Zucker und zeigt mikroskopisch nichts Krankhaftes.

Nervensystem: Das rechte Auge zeigt eine leichte Hornhauttrubung. Dic Pupillen sind rund, r. weiter als 1.; Licht- und Konvergenzreaktion geschicht bcidscits prompt. Konjunktival- und Kornealreflex ist vorhanden. Von seiten der Kopfnerven sind keine krankhaften Erscheinungen zu konstatieren. Reflexe: obcre Extremität normal. Der Patellarreflex ist beidscits deutlich herabgesetzt. Der Achillesschnenreflex ist r. schwach, l. mäBig auslösbar. Kloni bestehen nicht. Der Bauchdeckenreflex fchlt. I. besteht ein schwacher, aber deutlicher Babinski. Beide Beine sind hingegen schlaff gelähmt. Die: Muskulatur des Abdomens und beider Beine ist staik atrophisch, weich und schlaff. Das Gehen und Stehen ist unmög. lich. Dic Sensibilität ergibt folgende Störungen: vorn reichen beidscits dic Anästhesic und Analgesie bis zum 4. Interkostalraume, die Hypästhesic und Hypalgesic in einer schmalen gleichmäßigen Zone bis zum oberen Rande der 4. Rippe. Oberhalb derselben sind die Empfindungen normal. Hinten reichen rechts die Anästhesic und Analgesie bis zwischen den 6. und 7. Brustwirbcl, die IIypästhesic und Hypalgesie bis zum 4. Brustwirbel; links die Anästhesic und Analgesic bis zum 7. Brustwirbcl, die Hypästhesie und Hypalgesic bis zum 4. Brustwirbel. Dic Wi r be Is ä u l e zeigt keine Abweichung von der Norm. Achsendruckschmerz ist nicht vorhanden. Der 3. Brustwirbel ist auf starken Druck etwas schmerzhaft. Verschiedene Röntgenuntersuchungen ergaben stets negative Resultate. Die Lumbalpunktion lieferte einen klaren, stark gelbgefärbten I.iquor. Die Wassermannsche: Reaktion von Blut und Liquor ficl negativ aus. Die Nonnesche Reaktion war dagegen stark pos:tiv. I.ymphocyten waren im Spinalpunktat nicht worhanden, wenig rote Blutkörperchen waren nachweisbar. Die Temperatur schwankte жwischen 368 und 37.6.

Finige Tage vor der Operation wurden die Schmerzen zwischen den Schulterblättern stärker; dic Patientin lokalisicrte sie zwischen den 2. und 4. Brustwirbel. Ziemlich rasch trat dann dirckt links vom 3. und 4. Brustwirbel einc geringe längsovale, $3: 4^{1 / 2} \mathrm{~cm}$ messende Vorwölbung von fluktuicrender Konsistenz auf. Die neurologische: I)iagnose lautete auf Tumor in der Gegend des 3. Wirbels, der infolge der in wenigen Tagen entstandenen Iähmung zunächst 
für „malign, wahrscheinlich Sarkom", gehalten wurde. J)as Auftreten der fluktuierenden Vorwölbung dicht neben dem 3. und 4. Dornfortsatz gestattete jedoch die Diagnose cines Senkungsabszesses, als dessen Ausgangspunkt wir trot $\iota$ des negativen Resultates der Röntgenuntersuchung cine tubcrkulöse Erkrankung des 3 . oder 4. Wirbels annahmen.

Die Operation fand am 31. 1. 1917 statt (Prof. Kirschner). Zuerst wurde die Vorwölbung punktiert und mit einer dicken Kanüle ca. $35 \mathrm{ccm}$ gelblicher, mit nekrotischen, käsigen Bröckeln gemischter, für eine tuberkulöse Erkrankung typischer Inhalt entleert. Dann wurde unter I.okalanästhesie durch cinen vom 2. bis 6. Dornfortsat\% reichenden rechtskonvexen Bogenschnitt und durch Beisciteschieben der Muskulatur mit einem breiten Meißel die IVirbelsäule freigelegt. Blutstillung grö̉ßerer ( efäße durch Ligatur, der kapillaren Blutung durch Kompression. Der dicht links vom 3. und 4. Dornfortsat\% liegende kalte AbszeB wurde beim Ablösen der Muskeln miterïffnet. Er war kleinhühnereigroß, gegen die Umgebung durch eine derbe Kapsclmembran scharf abgegrenzt und mündcte durch das 1. dritte Foramen intervertebrale mittels cines nicht gan\% bleistiftdicken liortsatzes in den Wirbelkanal. Nach Austamponieren der Abszeßhöhle wurde der tuberkulöse Sack ('xstirpiert. Hieraut wurden mit der Lucrschen Zange die: Dornfortsätze: 2--4 entfernt und der Virbelkanal eröffnet. Die Dura mater zcigte nun, mit der Witte in der Höhe des 3. Wirbels liegend, rine s charf abgegrenztc, leicht höckerige Auftreibung in einer lä̈ngevon $5,7 \mathrm{~cm}$, in eincr Breite von I,I cm und in einer I)icke, b zw. Tiefe ron $1--1,3$ cm. Dor .granulomartige 'Tumor" sals jedoch nicht etwa cintach der l)ura auf, wie das gewöhnlich bci der von einer Caries ausgehenden epiduralen Tuberkulose der Fall ist, sondern Granulom und I) ura bildeten eine feste cinheitliche, nicht voneinander $z \mathrm{u}$ isolierende Masse. Das Tumorgebilde wurde der länge nach gespalten. Auf der Schnittfläche reigten sich deutlich gelbliche nekrotische Käseherde. Aus dem oberen Einde des Durasackes quoll schr reichlich, aus dem unteren Ende nur wenig klarer Liquor hervor. I m I3ereicheder f eschw ustbild ung a a r das Rückcnmark sattelartig ä Berst stark plat gedrückt und komprimiert (s. Fig. l u. 2). Im übrigen zeigten die weichen Iäute und das Mark keine abnorme Beschaffenheit. Mit ciner feinen geknöpften Schere wurde die greschwulstartige Bildung aus der gresunden 1)uraumgebung excidiert, so daB ein größeres Durafenster übrig blieb. Dic Wunde wurde im BC. reiche des Abszesses mit ca. $4 \mathrm{ccm}$ eincr 3 proz. wässerigen Pyoktaninlösung ausgetupft und hicrauf vermittelst durchgreifenden Muskel-Fascienknopfnähten aus Katgut mit Fascienzwischennähten geschlossen. IIautknopfnähte aus Scide. Stcriler Verhand. Sritenlage, da bei Bauchlage über starken J.uftmangel gecklagt wird. 
Am 12. II. wurden die Nähte entfernt. Mit Ausnahme einer kleinen Fistel, durch die liquor abfloß, war cine primärc IIcilungr eingetreten. Die Kopfschmerzen hatten abgenommen; Status im übrigen unverändert. I6. II. Die Wunde klaffte zum größten Teile. Sie war von Liquor durchtränkt. Im unteren Wundwinkel trübe, eitrige Sekretion. Temperatur stets zwischen 37 und 38 Grad. Die Wunde wurde mit mittelstarker l'yoktaningaze locker tampo. niert. 25. II. Die Kopfschmerzen hatten nachgelassen. Pat. fühlte

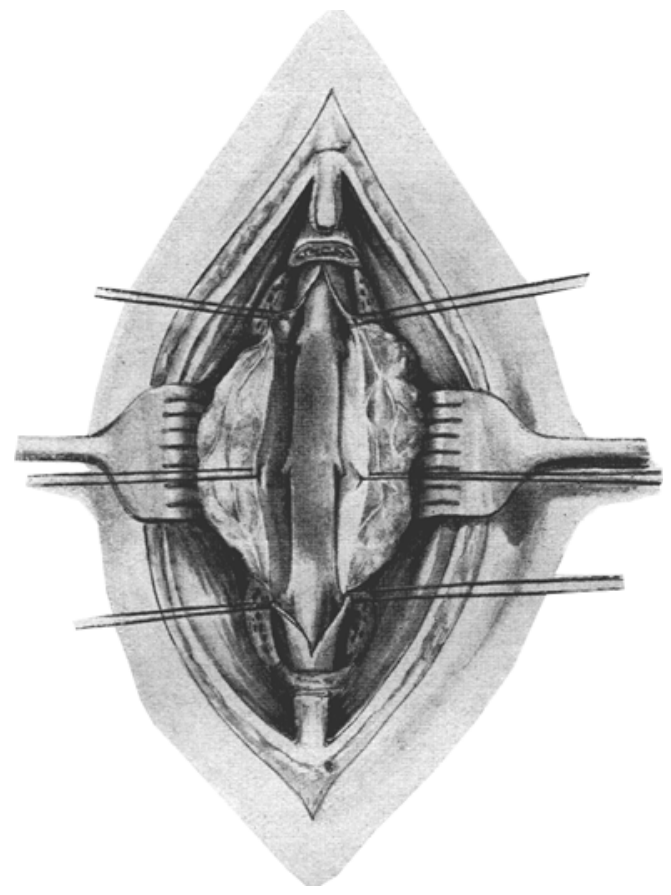

Fig. I.

Nach der Laminectomic wird die Dura gespalten. Man sieht die tumorartige Anschwellung der Dura und das infolge Kompression plattgedrücktc Rückenmark.

sich angeblich besser, glaubte auch in den Beinell ofter eine geringe: Empfindung zu haben. Obj. Status idem, kcine Besserung. Der Liquorfluß sistierte. Dic Wunde hatte sich unter Blaugazetamponads: vollständig̨ gereinigt und granulierte rasch zu. 30. Il. Status idem: es hatte sich eine Cystitis eingestellt. $\Lambda \mathrm{m}$ Sacrum, an den Fersen und unter den Schulterblättern begannen sich trotz Wasserkissen und sorgfältiger Hautpflege Dekubitalulcera zu entwickeln. Infolge der schweren Cystitis und der zunehmenden Dekubitalulcusbildung traten 
Temperatursteigerung und Verfall cin. Am 2I. III. crfolgte ohne jegliche Besserung der (2uerschnitsläsion der Exitus letalis. -

Auszug aus dem Scktionsprotokoll (Herr Prof. $\mathrm{K}$ a iserling): Mittelgroße lrau, in sehr dürftigem Ernährungszustande. An den Fersen, Schulterblättern, an den Strecksciten der Ellenbogen, sowie den Glutä:almuskeln ausgedehnte I)ekubitalulcera. Beide Pleurahöhlen sind infolge ausgedehnter bindegewebiger Ver. wachsungen zwischen den Pleurablättern vollkommen obliteriert. Herz: o. B. In der Spitze der 1. Lunge ist ein walnuBgroßer derber Knoten fühlbar. Auf dem Durchschnitt zeigt sich, daß dieser aus schiefrigem verhärteten Gewcbe besteht. Im übrigen ist die l. Lunge vollkommen lufthaltig. Sic erscheint auf $\mathrm{dcm}$ I)urchschnitt sehr blut-

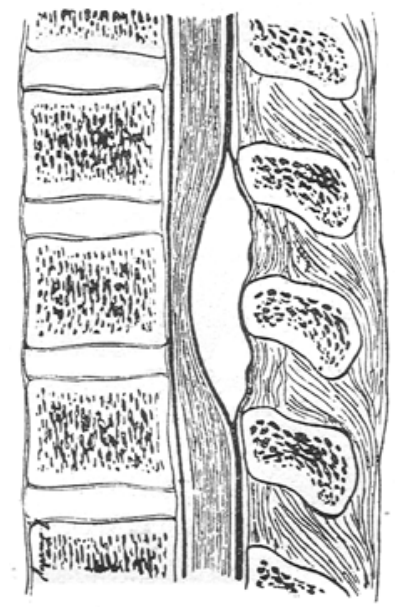

Fig. 2.

Schematische Darstellung der Ruickenmarkskompression durch den tubcrkulösen Granulationstumor der Lura.

reich. Die r. Lunge verhält sich ebenso, nur dab sich in der I,ungen. spitze statt des cinen großen mehrere etwa erbsengroße schicfrige, verhärtete Knoten finden. Mi'z, Pankreas, Nebennieren, Nieren o. B. In der Harnb'asc beiinden sich etwa Ioo $\mathrm{ccm}$ stark getrübten Urins. Die Harnblasenschleimhaut ist teils von schmierigem, schmutzig. grünlichem Aussehen, teils ist sie mit miBfarbigem Belage bedeckt. Genitalien, Leber, Verdauungstraktus o. B.

A nat. Diagnose: Tuberculosis durae matris spinalis (Höhe 3. Brustwirbel).

Dic sorgfältige makro- und mikroskopische Entersuchung der ganzen Iimgebung des I) uraherdes (inkl. der bei der Operation entfernten Knochenteile) hat kcine krankhaft'n Veränderungen crgeben. 
Vor allem konnte cine primäre tuberkulöse Lirkrankung der Wirbelsäulc oder des Rückcnmarkes ausgeschlossen worden. Nit Ausnahme. der kleinen schicfrigen, verhärteten Knoten in beiden I.ungenspitzen war nirgends in körper ein Krankheitsherd nachweisbar. Die histologische lintersuchung der erkrankten Dura hat folgendes ergeberal:

Die Dura ist srößtenteils kernarm. Dic einzelnen (iewebslagen sind zum Teil durch kleine Spalten getrennt. In der äußeren IIälfte der Dura findet sich cine Rundzelleninfiltration, die ohne scharfe Begrenzung in ein zellreiches Granulationsgewebe übergeht. In diesem Granulationsgewebe fincten sich grobe Inscln totaler kerniger Gewebsnekrose und zahlreiche typische Fipitheloid- und Rundzellentuberkel mit reichlichen I.anghansschen Riesenzellen. Fine strenge Abgrenzung des tuberkulösen Granulationsgewebes šcgenüber der Dura ist nicht nachweisbar; vielmehr wuchert es zwischen die Duralamellen hinein, grenzt an einzelnen Stellen unmittellbar an die Dura. außenseite und ist an wiederum anderen Stellen durch cinen schmalen Wall von mit Rundzcllen infiltriertem fibrösen Gewebe von der Dura getrennt. Dic Innenfläche der Dura durchbrechen die tuberkulöscn Granulationen nirgends.

Wenn schon dic sekundäre Tuberkulose der Dura mater operativ cine gewisse Seltenheit ist, so ist der hicr beobachtete Fall einer primären oder richtiger isolierten Duratuberkulose ein bisher -.. soweit ich feststellen konnte ... überhaupt noch nicht beschriebenes Krankheitsbild. Dic sekundäre Tuberkulose der Dura spinalis entsteht selten im $\Lambda$ nschluß an eine luberkulose' der Pia-Arachnoidea spinalis, die ihrerseits wieder entweder hämatogen oder öfter im Anschluß an eine tuberkulöse Meningitis cere bralis entstanden ist. Zumeist tritt die Tuberkulose der Dura mater spinalis vielmehr im Anschluß an cine Wirbeltuberkulose auf und stellt sich als cine chronische tuberkulöse Entzündung der 1)uraAußenfläche dar. Sie überzieht tumorartig in oft großer Ausdehnung die AuBenseite der Dura mit ciner bald mehr derberen, bald weicheren käsigen Granulationsgeschwulst. In der Regel beschränken sich diese Granulationen auf die AuBenseite der harten Haut. Nur selten wuchern sie, bilden in der Dura Tuberkclherde oder durchdringen sie vollkommen und erzeugen auch auf ihrer Innenscite Auflagerungen. In einem derartigen Falle kann sich cine sog. Pachymeningitis interna tubcrculosa hacmorrhag. oder eine miliarc Tuberkulose im Durasack entwickeln.

Die klinischen Erscheinungen treten in der Regel 
ərst dann deutlich hervor, wenn das Rückenmark selbst oder die uustretenden Wurzeln in Mitleidenschaft gezogen werden. Die Be:eiligung der Narkes kann bestehen in ciner zirkumskripten Meningitis mit Quellungs\%uständen oder Infiltrationen, die vom Rande der Medulla aus sich in die Substanz hinein verbreiten (meistens den Septen und Gefälßen folgend). Oder es treten im Mark Erweichungsherde auf, dic auf eine Ischämie durch Gefäßverschluß zurückgeführt werden. Ind schließlich können sich in der Rückenmarkssubstanz selber oder im Wurzelgebiet echte Tuberkel ausbilden; es handelt sich dann um die sog. echte tuberkulöse Meningo-Myelitis bzw. Meningo-Neuritis. Im Gegensatz zu den Ausfallserscheinungen, die durch F or ts chreit endes K rankheitsprozesses von den Rückenmarkshäuten a uf das Mark sclbst hervorgerufen werden, stehen die Störungen, die infolge Kompression des Rückenmarks durch den zwischen Mark und knöcherner Spange sich ausbildenden tuberkulösen „Granulationstumor" ausgelóst werden.

Die Diagnose einer auf die Dura mater spinalis selbst lokalisierten Tuberkulose wird vor der operativen Freilegung wohl niemals gestcllt werden können. Sclbst bei der Operation sah unser Fall in allem einem epiduralen sekundären tuberkulösen Granulationstumor gleich, wie er als Folge ciner tuberkulösen Wirbelerkrankung aufzutreten pflegt. Erst durch genaue Inspektion des brcitcn Operationsfeldes und durch die mikroskopischen Untersuchungen konnte cin anderer lokaler primärer Herd mit großer Wahrscheinlichkeit ausgeschlossen werden. Die Nachuntersuchungen bei der Sektion bestätigten dann mit Sicherheit das Fehlen jeder anderen lokalen tuberkulösen Erkrankung. Gewöhnlich wird man ir: der Differentialdiagnose zwischen tuberkulöser Spondylitis und Tumor schwanken. Die Differentialdiagnose ist oft schwieriger, als es in den Lehrbüchern dargelegt wird. Oppenheim ist in scinem Referate auf dem Budapester internat. medizin. Kongreb im Jahre Igog auf diese Differentialdiagnose nicht näher eingegangen und erwähnt nur ihre Schwierigkeit. Auch A ue r ba ch berichtet, daß die Differentialdiagnose zwischen Spondylitis tuberculosa und Tumor in viclen Fällen trotz eingehender Berücksichtigung aller diagnostischen Momente nicht immer mit absoluter Sicherheit zu stellen ist; ,insbesondere lasse man sich durch das 
Uberwiegen der motorischen und muskeltrophischen Störungen über dic sensiblen nicht von der Annahme einer Geschwulst abhalten". Im Falle eines Zweifels rät er dringend zu cincm chirurgischen Eingriff. In unseren Falle wurde von den Neurologen wegen der in wenigen Tagen eingetretenen Lähmung cin maligner 'lumor, ,wahrscheinlich Sarkom", diagnostiziert. Da sich aber ein paar Tage vor der Operation dic fluktuierende Vorwölbung neben dem 3. und 4. Brustwirbel gebildet hatte, glaubten wir, cine Tuberkulose annehmen zu müssen. Das Auftreten eines Senkungsabszesses oder die Entstehung einer Fistel klärt die Situation na. türlich stets mit einem Schlage. Sonst ist zur Differentialdiagnose viclleicht noch zu verwerten, daß bei Wirbeltuberkulose im Frühstadium dic Extension oft cine Erleichterung bringt, während sic bei Tumoren fast immer die Beschwerden und die objektiven Symptome verschlimmert (A u e r b a ch). In jedem Falle sind wir cbenso wic die meisten Neurologen der Ansicht, daß bei zunchmenden, auf eine lokalisierbare Querschnittslähmung hinweisenden Rückenmarkserscheinungen, bei Ausschlu\} ron Lues, eine sofortige explorative Laminektomie notwendig ist. Wir können dies um so cher verantworten, als der Eingriff durch die von uns stets benutzte Lokalanästhesic scine Hauptgefahren, die der Blutung und die der postoperativen Lungenerkrankungen, verloren hat. Bei frühzeitig vorgenommener Operation hat man dic Aussicht, den Kranken zu heilen, während nach unserer Erfahrung die s päte, nach längerem Bestchen ausgedehnter I.ähmungen ausgeführte Operation schlechte $A$ ussichten hinsichtlich des Rückgangs der Querschnittserscheinungen bietet. Es ist durchaus verfehlt, kostbare Zcit zu verlieren, um die Möglichkeit einer sicheren Diagnose über die $A r t$ des raumbeengenden Prozesses abzuwarten. Es soll operiert werden, sobald die topische Diagnose eines raumbeengenden Prozesses gestellt ist.

\section{Literaturverzeichnis.}

A uerbach, Lber operative Neurologie. Bruns' Bcitr. z. kl n. Chir. IgII, Bd. 74.

$A$ uerbach und Brodnitz, Cber cinen groben intraduralen Tumo des Cervikalmarkes, der mit Erfolg exstirpiert wurde. Gren/gebiete I905, Bd. 15. 
Goldscheider, leber Myelomeningitis cervicalis anterior bei Tuberkulose. Berl. klin. Wochenschr. I8y I.

(; unsser, beitrag zur Kenntnis der Rückenmarkstubcrkulose. Inaug. Diss. Tübingen, 1890 .

Il a scorec, J.., contribution à l'étude de la tuberculose de la moeile Épinière. Arch. de Neurol. T. XXX, 1895, Mr. ro3.

II enselhen, F.. Nultiple Fndotheliome der Jura spinalis in Bercich cinci Pachymeningitis tubcrculosa. Zieglers lieitr. z. patholog. Anatomie แ. z. allg. Pallologic, I9ro, Bd. 49.

Il jl debratnd, "Rückemmarkschirurgie". Verhandl. d. Deutsch. Gescllsch. f. Chirurgit: Ig)10, S. I9.

II iiller, I. D., Ein Fall von solitärer Tuberkulose des Rückenmarkes, zugleich ein Beitrag zur Lehre von der B Bown-S c q u r d schen IIalb. seitenlähmung. Deutsche Zcitschr. f. Ncrvenheilk. I898, Bd. I 2.

() p penheim, Bciträge zur Diagnostik und Therapie der Ceschwülste im Bereich des zentralen Nerrensystems. Berlin, 1907.

, I)iagnose und Behandlung der Geschwülste innerhalb des Wirbel. kinals. Deutsche med. Wochenschr. 1909, Nr. 44.

Scarpatteti, Befund ron Kompression und Tuberkel in Rücken. mark. Jahresher. f. Psychiatrie, Bd. I5, S. $31 \mathrm{I}$.

Sihlesinger, Tumoren der Rückenmarkshäute. Beitr. z. Klinik der Rückenmarks und Wirbeltumoren. Jena 1898 .

Si hma us. I)ic Kompressionsmyelitis bei Karics der Wirbelsäule. Wies. baden 1890 .

ثtursberg, Die operative IBehandlung der das Rückenmark und die Caluda çuina komprimicrenden Noubildungen. \%entr. f. d. Gremz. gebiete, 1908, Bd. 11.

te iegler. Lehrbuch d. Path. u. path. Amatomic. 1892, 7. Aull. 